\title{
Evolving Mechanisms of Morphogenesis: on the Interplay between Differential Adhesion and Cell Differentiation
}

\author{
P. HogeweG* \\ Theoretical Biology and Bioinformatics Group, Utrecht University, Padualaan 8, 3584 CH Utrecht, \\ The Netherlands
}

(Received on 1 February 1999; Accepted on 5 January 2000)

\begin{abstract}
Differential cell adhesion, mediated by e.g. integrin and cadherins/catenines, plays an important role in morphogenesis and it has been shown that there is intimate cross-talk between their expression and modification, and inter-cellular signalling, cell differentiation, cell growth and apoptosis. In this paper, we introduce and use a formal model to explore the morphogenetic potential of the interplay between these processes. We demonstrate the formation of interesting morphologies. Initiated by cell differentiation, differential cell adhesion leads to a long transient of cell migrations, e.g. engulfing and intercalation of cells and cell layers. This transient can be sustained dynamically by further cell differentiation, and by cell growth/division and cell death which are triggered by the (also long range) forces (stretching and squeezing) generated by the cell adhesion. We study the interrelation between modes of cell differentiation and modes of morphogenesis. We use an evolutionary process to zoom in on gene-regulation networks which lead to cell differentiation. Morphogenesis is not selected for but appears as a side-effect. The evolutionary dynamics shows the hallmarks of evolution on a rugged landscape, including long neutral paths. We show that a combinatorially large set of morphologies occurs in the vicinity of a neutral path which sustains cell differentiation. Thus, an almost linear molecular phylogeny gives rise to mosaic evolution on the morphological level.
\end{abstract}

(C) 2000 Academic Press

\section{Introduction}

It is well known that cell adhesion is important in development. As it is put in a review by Gumbiner (1996): "Cell adhesion systems should be regarded as a mechanisms that help translate basic genetic information into the complex three dimensional patterns of cells in tissues". Cell adhesion molecules, cadherins and integrins, among which there is much cross-talk (Monier-Gavell \& Duband, 1997), do appear to be nexus points

\footnotetext{
*E-mail: ph@binf.bio.uu.nl
}

in cell-signalling pathways, including those of cell growth factors, and their expression and modification are intimately related to cell differentiation, cell mobility, cell growth and cell death (for review see also e.g. Howe et al., 1998; Marrs \& Nelson, 1996; Giancotti, 1997). Interestingly, it also has been shown that cell volume changes by themselves can trigger cell growth and cell death in endothelial cells (Chen et al., 1997; Ruoslahi E., 1997), and that only quantitative differences in cadherin expression can cause cell sorting, tissue spreading and specific spatial patterning (Steinberg \& Takeichi, 1994). Gumbiner (1996) 
concludes his review with: "This coupling between physical adhesion and developmental signalling provides a mechanism to tightly integrate physical aspects of tissue morphogenesis with cell growth and differentiation, a coordination that is essential to achieve the intricate patterns of cells in tissues". This is indeed the focus of our study.

To this end, we propose a new modeling approach to study the interplay between, and the coordination of, processes at different organizational level; here (differential) cell adhesion and cell differentiation, and the resulting cell migration, cell growth/division and cell death. In this modeling approach, we minimize prior assumptions and parameters. Thus, in contrast to other abstract models on morphogenesis and pattern formation, which abstract to a minimum process at one level, we abstract to a minimum process connecting different levels of developmental systems.

We use an abstract receptor-mediated differential adhesion process as the core of the model. This process connects all the other levels in our model. Gene regulation networks define differential adhesion between cells, differential adhesion changes size and shape of cells and therewith create long-range forces between the differentiated cells, and there-with cell migration, and mechanical stresses on cells. These mechanical stresses trigger cell growth, division and death. Cell migration, cell division and cell death alter cell contacts and therewith, via inter-cellular signalling, modify gene expression, and thus differential adhesion. Note that although in biotic systems the complex pathways involved in the expression and modification of cell adhesion molecules, (inter)cellular signalling, cell growth, cell cycle control, cytoskeleton reorganization, etc., appear to allow for all possible couplings between these processes, we restrict our model to cases where these pathways ultimately lead to relaxing physical stresses generated by differential adhesion. (Thus in our model, and in accordance with the experimental results cited above, cell stretching will lead to growth/division and cell squeezing to cell death rather than the other way around.) We study to what extent the relaxation of stresses will be sufficient to generate interesting morphogenetic processes.
However, notwithstanding this simplification, the set of possible gene-regulation networks, inter-cellular signalling processes and cell adhesion parameters is of course potentially enormously large, and we do not expect that all of these (or even many of these) will exhibit interesting morphogenetic processes. Indeed for most biological processes this is the case: a complex and specific microscopic organization gives rise to macroscopic processes, and perturbation of the specific micro-structures most often cause deterioration. Some macroscopic processes may nevertheless be describable in terms of relatively simple microdynamics, but this will be at the cost of severing the automatic connection between these macroscale phenomena and others (at a macroscopic or microscopic level) generated by the underlying complex microscopic structure of a biotic system. In my view, a major challenge to theoretical biology is to develop modeling approaches to study the coordination between processes at different scales, and to permit modeling of relatively high complexity and specificity at the microscopic level, but at the same time adhere to the methodological important minimization principles. This is what is attempted in the research reported here.

Our approach (see also Hogeweg, 1998) is to use an (artificial) evolutionary process to derive instance of specific (model) structures at the microscopic level. The selection criterion used in the evolutionary process in the study reported here is cell differentiation: we maximize the distance between the different attractors of the gene regulation network attained by cells in the developing structure. Note that this fitness criterion (1) does not reflect "realistic" evolutionary pressures and (2) does not include the features in which we are interested, i.e. morphogenesis. The latter choice of fitness criterion would trivialize the results ("we will get what we ask for"). The former, if it could be done at all, would force us to extend the model too far from our focus, i.e. morphogenesis ("no one lives by shape alone"). Instead, we take as a fitness criterion something which is a pre-requisite to the focus of the study: here, without cell differentiation no shape changes are to be expected. Thus, our results will be of the form: "gene regulation networks, selected for cell differentiation, exhibit such and such modes 
of morphogenesis by means of locally relaxing forces generated by differential adhesion between cells".

We will slow that these modes of morphogenesis which occur as side-effects of cell differentiation include gastrulation-like engulfing, meristematic growth, intercalation and stretching, and very complicated orchestration between cell growth, cell death and cell differentiation, which dynamically cause pseudo-isomorphic outgrowth. Moreover, we show that combinations of these processes in different parts of a developing "critter" may have long-range interactions, so that a combinatorial explosion of morphologies is possible.

Thus, the resulting morphogenesis resembles processes observed in biological systems. The model suggests that these morphogenetic processes are the ones which are easy in systems governed by the interplay between cell adhesion and cell differentiation. Their occurrence in biotic systems does not need an explanation in terms of specific optimality criteria governing the evolution of morphogenesis in biological systems, nor do they need coordination between cell migration, cell growth and cell death beyond those generated from local relaxation of stresses generated by differential adhesion, although this is mediated in biotic systems through complex signalling pathways. One should be aware that other types of micro-dynamics, e.g. those based on gradients, intra-cellulary regulated cell shape changes, or others may prove to be sufficient as well. If so, this would only strengthen our conclusion that these types of morphogenesis are "generic".

\section{The Model}

The model includes evolution, development and gene regulation, which mutually define each other. In evolutionary time gene regulation networks evolve. In developmental time cell divisions take place, and the evolved gene regulation networks plus "maternal signals" lead to differential gene expression and therewith to differential adhesion between cells. Through differential adhesion, cells can come into contact with other cells which can lead to further cell differentiation. Finally, the fully developed "critter" is assigned a fitness on the basis of the amount of difference of gene expression in cells. This drives the gene network evolution. Finally, we study the critter's shape (i.e. morphogenesis ss), i.e. a feature not included in the fitness criterion.

\subsection{INTER-CELLULAR INTERACTIONS: DIFFERENTIAL ADHESION}

The core of the model is the two-scale asynchronous CA-like model for cell adhesion as first proposed by Glazier \& Graner (1993). In this model, a biological cell is represented in many cellular automata (CA) cells having an identical state (i.e. the cell identification). The CA update rules are such that a CA cell takes the state of a neighboring CA cell if this reduces "surface energy", subject to a volume-conservation constraint, i.e.:

$$
\begin{gathered}
H=\sum \frac{J_{i j}}{2}+\sum J_{i m}+\lambda(v-V)^{2}, \\
P_{i->j}=1 \quad \text { iff } \quad \Delta H<-0.1, \\
P_{i->j}=\mathrm{e}^{-(\Delta H+0.01)} \quad \text { iff } \quad \Delta H \geq-0.1,
\end{gathered}
$$

where $H$ is the total surface bond "energy", summed over all cell-to-cell contacts and cell media contacts, and the size conservation over all cells. $\Delta H$ is the change in $H$ if the site under consideration is changed from $i$ to $j$, i.e. cell $j$ extends itself into the area previously occupied by cell $i$. $P_{i->j}$ is the probability that this change will take place.

Thus, the behavior (movement) of a cell is the result of extending and retracting its cell membrane, and this process is governed by surface receptors which define cell adhesion. Glazier and Graner showed that this can lead to cell sorting, which is quantitatively in agreement with that shown in retina cell in vitro. The beauty of the model is that it can easily be extended to include other processes. For example, Savill \& Hogeweg $(1997 \mathrm{a}, \mathrm{b})$ introduced

- Chemotaxis (probability of copying depends also on some gradient), and used this in a model of Dictyostelium development "from 
single cells to crawling slugs" (see also Marée et al., 1999; Jiang et al., 1998),

- Food-induced cell growth and division.

In the current model we introduce and use:

- Cell death due to squeezing: depending on the surface bond energies $J$ and the non-elasticity $\lambda$ a cell may be reduced to zero volume, i.e. it may die.

- Cell growth due to stretching: when the actual size of a cell $(v)$ exceeds the target size $(V)$ plus a threshold $(\tau)$ the target volume is increased $(V=V+1)$. (In the reported experiments cell growth was not used during evolution, but interesting evolved critters were studied with and without such growth.)

- Volume-triggered cell division: when $V$ has become twice the reference cell size the cell divides. Division is in the middle of and perpendicular to the longest cell axis.

These assumptions are in agreement with experiments which have shown that cell volume alone can indeed trigger apoptosis and cell growth/ division (Chen et al., 1997; Ruoslahti, 1997), and in vitro experiments which have shown that the positioning of microtubule asters is such that the cell will divide perpendicular to the longest axis (Holy et al., 1997).

\subsection{GENE REGULATION, INTER-CELLULAR SIGNALING AND CELL DIFFERENTIATION}

Gene regulation is represented in the form of a boolean network of, in the experiments reported here, 24 nodes and a connectivity $\leq 2$. Boolean networks were first used for studying cell differentiation by Kauffman (1969); Jackson et al. (1986) demonstrated spatial patterning in tissues of cells regulated by boolean networks. In our experiments, the network topology and the boolean functions are initially generated randomly, with an equal probability for each of the 16 two-input binary functions, and a 0.5 probability of an input from another gene (otherwise it gets an all-zero input). They are subsequently subject to mutation and selection (see below).

Of the 24 nodes (genes), ten are used to define the inter-cellular adhesion [expressed as the parameter $J_{i j}$ as defined above in eqn (1)]. Five of these serve as "locks" and five as "keys", with complementary bit-matching:

$$
J_{i j}=\frac{1}{2}\left(\sum_{k=0}^{4}\left(M_{k}^{i j}\right)^{k}+\sum_{k=0}^{4}\left(M_{k}^{j i}\right)^{k}\right),
$$

where $M_{k}^{i j}$ is the matching of the $k$-th key of cell $i$ in the $k$-th lock of cell $j$. It is done in this way to allow all possible adhesion parameters also between identical cells. The "adhesion" with the medium is defined as

$$
J_{i, m}=\sum_{l=0}^{4}\left(N_{l}^{i}\right)^{l},
$$

where $N_{l}^{i}$ is the state of the $l$-th node of cell $i$, and $l$ overlaps half with the "locks" and half with the "keys" defining inter-cellular adhesion.

Note that the cell adhesion is cell state dependent and therefore can change over time.

In addition, two of the adhesion-defining nodes are also used for inter-cellular signaling, i.e. a node of the boolean network can have input from these genes of neighboring cells rather than itself. The inputs of all neighboring cells are combined into the environment vector by an OR function. We model in this way the role of cell adhesion molecules in inter-cellular signaling. In biotic systems, adhesion strength can be modified by peripheral processes, e.g. receptor clustering. Note that, although we interpret our boolean networks verbally as gene-regulation networks, they may partly model peripheral processes as well.

The "zygote", with a pre-defined network, is initiated in state 0 (no genes expressed) and with an all 0 environmental vector (no neighbors). At every time-step the boolean network is updated (synchronously) and local cell membrane movements governed by cell adhesion are executed (asynchronously). The first seven cell divisions are pre-scheduled, and all cells divide simultaneously. Both daughter cells inherit the state of the network of the mother cell. However, after the first and the second cell divisions unequal cell division can occur. This is done by flipping the state of a pre-defined node during 1 time-step in one of the daughter cells. We model herewith crudely a "maternal factor", which can induce cell differentiation. Note that only a small subset of 
all possible networks will "use" this signal, and often only one of the two signals is used in the evolved networks. Further cell differentiation occurs through "induction" by neighboring cells.

Note that by using such maternally induced unequal cell division we incorporate a wellknown biological feature, but deviate from most modeling efforts which focus on inducing initial differentiation from an almost homogeneous state [e.g. Turing-type models, isologous diversification (Kaneko \& Yomo, 1997), or on discrete differentiation starting from a continuous gradient]. Here we are interested in how initial differentiation leads to morphogenesis.

It is well known that synchronous boolean networks often have many attractors. Note that in studying development, we do not start at multiple initial conditions, so that a network with many attractors often fails to exhibit cell differentiation in this setting. On the other hand, multiple attractors of the intra-cellular gene regulation network are not necessary for cell differentiation. For example, out-of-phase oscillations in the same attractor can lead, via the cell signaling mechanism, to different (fixed point or limit cycle) attractors which are induced and maintained by the cell contact. In the latter case, we have cell differentiation, but no, or limited, cell memory as cells de-differentiate in isolation (re-instantiating cell contact may, or may not re-establish the same differentiation pattern). Obviously, differentiation with "memory" (i.e. having a stable intracellular attractor) and without memory can be superimposed. We will see that different modes of morphogenesis need different memory properties of the differentiated cells.

\subsection{EVOLUTION}

We use a simple Genetic Algorithm for the evolution, i.e. a population of regulation networks compete for a pre-defined fitness criterion. This fitness criterion is the amount of difference in gene expression among all cells (measured as the Hamming distance between the average cell state in its attractor). It is measured over a limited period of time in a 128-cell "critter". The minimum value seen in that time is taken as the fitness.

This fitness criterion is used because having different gene expression patterns is a pre-condi- tion for "interesting" development and most networks do not lead to cell differentiation. It is not meant as "natural" fitness criterion or as an hypothesis about the evolutionary pressures leading to multi-cellularity. Moreover, it is not the case that the critters with most cell types exhibit the most interesting behavior. In fact, in the experiments shown here, we add the constraint that only fixed-point attractors or short cycles (period 2-3) contribute to the fitness because, without this constraint, differentiation initiated by phase differences of long state cycles (cf. Kaneko \& Yomo, 1997; Furusawa \& Kaneko, 1998a,b) is too "easy" and leads to too many different cell types (attractors) and therewith appears to inhibit coordinated development. Nevertheless, the criterion as used fulfills our needs because it enables us to focus on interesting morphologies without many a priori constraints.

Point mutations, including changes in connection and in boolean function, are the only genetic operator used (no crossing over, gene duplication, etc.). Initially, as long as none of the networks shows differentiation, new networks are generated randomly.

\subsection{MODEL INITIATION, MODEL PARAMETERS AND DIFFERENCES IN EVOLUTIONARY OUTCOMES}

Table 1 gives an overview of program structure of the evolutionary model. The evolutionary model is initiated with a population of random gene-regulation networks. The results presented in this paper were all obtained with about the same model parameters, see Table 1 . The differences between the evolutionary outcomes depend on the random initial population as well as randomness is selection, and even scheduling, in particular in the initial stages of an evolutionary run. During evolution cell growth/division was disabled. Pictures of all evolved critters were stored. The development of selected critters was studied interactively, for longer developmental times then those used in the evolutionary run and with cell growth/division enabled.

\section{Developmental Dynamics}

In this section, we first describe five examples of developing critters, and characterize the 
TABLE 1

Overview of evolution and development model



on main processor:

Reproduction and evolution

RECEIVE fitness and network of processor $p$, store with network in position $p$

Selection choose the highest fitness of 7 random choices out of population)

Mutation copy chosen parent and mutate $(\mu=0.5)$ inputs or boolean functions.

SPAWN new network to subprocessor $\mathrm{p}$

Note: The model runs parallel in real time (Using PVM).

Because of scheduling differences no two evolutionary runs are identical.

observed development in terms of determinism and randomness, cell- and environment-based memory, long-range interactions, and selfenhancement of transients and frustration.

\subsection{SOME EXAMPLES}

In Fig. 1 we present some examples of the zoo of critters which were evolved. It is clear from the figure that a great diversity of morphologies evolved. Note again that evolution operates solely on gene regulation and no evolutionary pressures related to shape were present. The way in which the various shapes emerge from the "egg" and develop is striking.

Engulfing. Figure 1(a) shows an elaborate engulfing process which phenomenologically resembles "gastrulation". Engulfing itself is a very basic property of differential adhesion. Glazier \& Graner (1993) already showed that celltype A engulfs celltype B if $J_{a b}<J_{m b}$ and $J_{a m}<J_{b m}$ ( $m$ is medium). Indeed, simple engulfing is seen in many evolutionary histories, see e.g. Fig. 3(c). In the particular case shown in Fig. 1(a) engulfing operates in consort with cell death and cell (re)differentiation, and cell growth/division, and so forms a (hollow) 
structure with cells of the same cell lineage on the inside and the outside. It does so with only four different gene expression patterns (all fixed-point attractors). Cell growth and division affect mainly the engulfing cell, and are needed to have enough of these cells to "go around". Precise timing of the various processes is needed: slight variations lead to different morphologies [see Fig. 2(a)].

Budding and elongation. Figure 1(b) shows an outgrowing "stem", from a small initial bud, again with only one stably differentiated cell lineage, which induces neighborhood-dependent differentiation in the other cell lineage. The elongation occurs because of a "frustrated" tendency of the gray cells to engulf the yellow cells, which have high $J$ with the medium. Yellow cells occur at both ends. Engulfing cannot happen because yellow and gray cells re-differentiate into each other, dependent on contact with the blue cells (which intercalate into gray cells). Without cell growth/division, the stem breaks and 2 blobs remain, with cell division the elongated stem persists indefinitely.

Morphogenetic dynamics, cell growth and cell death. Figure 1(c) shows the formation and dynamic maintenance of complex asymmetrical structures due to a precise orchestration of cell death, cell differentiation and cell growth/ division. Maternally induced differentiation is into long 14-point cycles, from which the fixedpoint "adult" cells arise in a context-dependent way. Initial shape changes happen because red and gray are condemned to be neighbors (again because of the neighborhood-dependent redifferentiation) but have high mutual J. Figure 2 shows that continued apoptosis and cell growth/division do occur and are needed to maintain the complex structures.

Intercalate and stretch. Figure 1(d) shows again the formation of a long protrusion, but by another mechanism, which can be described as "intercalate and stretch". The interface between brown and blue cells increases because of intercalation and redifferentiation of the green ones. Simultaneously, the brown cells push into the flexible green cells ( $J_{\text {green }}$, green is low) and thus narrow the contact zone between the blue and the green cells. This leads to the protrusion of a width of 2-3 cells. However, once the blue cells are on either side the protrusion contracts again.
An interesting feature is the "gliders" along the edge of green cells. They consist of a member of all three stably differentiated maternally induced cell lineages. The one of the "brown" cell lineage (white) pushes the "blue" one (purple) along the green-derived yellow ones. These gliders often initiate and speed up the contraction. Moreover, they make the final structure very dynamic, "thrusting" around the green cells. The described scenario is independent of the occurrence of cell growth/division, which mainly leads to an increase of the light-green cells.

Meristemes and intercalation. Figure 1(e) shows the most complicated case with many different gene expression patterns ( 24 all through development), derived from three stably differentiated cell lineages due to the two maternal signals. The further differentiation is neighborhood based, and induction of one new cell type again induces next ones. There is extensive cell migration due to engulfing, intercalation and invagination, leading to creation of new gene expression patterns, and loss of them as well. A representation of the distribution of the cell lineages in a late developmental stage is given in Fig. 3(c). Strong meristematic growth which occurs in the upper part, is governed by three neighborhood differentiated layers of cells all belonging to the same cell lineage. The lower ones are trying to intercalate and engulf the upper ones, but differentiate into them when successful. The "curl" develops around an isolated cell of another cell lineage because contact with that cell lineage defines the lower (green) differentiation pattern. Stretching due to intercalation of stably differentiated cell lineages occurs in the rear and the combination of this stretching to the rear and re-differentiating cells in the top leads to the "neck" separating the top and the rear part [see Fig. 3(c) for experimentally modified morphology]. Without cell growth the stretching leads to breakup of the structure. We discuss the evolutionary sequence which produced this criter in Section 4.

\subsection{DETERMINISM AND RANDOMNESS}

No two critters are identical, not even those with identical genomes, which are developed with the same global parameters $(\lambda, T, \tau)$. Nevertheless, each critter is clearly "itself" throughout 
development, and individuals with functionally the same genome are clearly recognizable as such.

The differences arise because randomness in cell-wall movement can be amplified by cell differentiation, and by the dependence of the division plane on cell shape. Such amplified random differences lead in the above-discussed examples to:

(a) Changes in the engulfing pattern. The engulfing cells may fail to get inside before closure, or closure may fail to occur, so that a "gut" is formed (but it is not opened on the other side) [Fig. 3(a)].

(b) The initial occurrence of no or more than one yellow bud. When more buds are formed there is global competition between buds and only one prevails. The competition phase slows down the elongation, but the end result is very similar. This is true for the "native" structure.
Global competition is less strong in experimentally manipulated ones without intercalation of the blue cells, and two buds may persist and grow (data not shown). When no bud happens to form, no elongation occurs.

(c) The initial differentiation pattern is extremely sensitive to noise, and therefore the critters may have quite different shapes [cf. Fig. 1(c) and Fig. 2]. Nevertheless in later stages the initial shape is further enhanced, and rather independent of noise.

(d) Initial development is rather insensitive to noise. The formation of the protrusion always occurs in approximately the same manner. It may however break when a very thin bridge of brown cells happens to form. Broken parts round off relatively rapidly, blue cells surrounding the brown ones.

(e) Although some timing differences do occur, the later stages look very similar in all cases.

FIG. 1. Examples of morphogenesis. The developmental dynamics is shown of five evolved "critters". They exemplify different morphogenetic mechanisms. (a) Cell migration and engulfing four fixed-point attractors. The purple cells are stably differentiated by unequal cell division at four-cell stage. They cause neighbor-based differentiation of pink cells into green ones. Moreover, they engulf the cell clump. The pink cell-clump falls apart due to high mutual $J$ values, and subsequently die off unless "captured" by contact with other cells. The olive cells are of the purple lineage, but not in contact with others of that lineage; they have other adhesions, but the same induction properties as the purple ones. Cell growth mainly of purple ones initially on top, later also at the inside. $J(i j)=5,11 ; 21,11,11 ; 8,-, 20,21 ; 8,-20,-,-,(i=$ purple, green, pink, olive: $j=$ medium $, \ldots, i)$. ( - : not in use because of redifferentiation) $\lambda=0.5, \tau=2, T=4$. (b) elongation and budding. four fixed-point attractors. The blue cells are stably differentiated by unequal cell division at four-cell stage. They cause neighbor-based differentiation of yellow cells to gray cells. Gray cells engulf blue cells rapidly. Blue and gray cells intercalate slowly. Formation of small yellow bud from gray cells which engulfed blue cells, but lost contact with blue cells leads to elongation. Growth takes place along edge of yellow and gray cells and distributed over the stem by stretching [see Fig. 3(b)]. Green cells are of the blue cell lineage but not in contact with it, and play no role in developmental dynamics. $J(i j)=24,7$; $4,5,4 ; 12,-, 8,8$ ( $i=$ blue, gray, yellow; $j=$ medium, $\ldots, i) ; \lambda=0.5, \tau=5 ; T=3$. (c) Cell death redifferentiation. three fixed-point attractors, plus several limit cycle attractors in early developmental stages (pink, blue) and as isolated cells in later stages (green). No stable cell memory-based differentiation. Light gray and red cells mutually induce and other, but have high $J$ values with each other. Cell death mainly due to loss of volume of isolated dark gray cells, which differentiated from red cells on loss of contact with light gray cells. Cell growth of red and light gray cells. Green cells differentiate to red cells or gray cells dependent on cell cycle stage on contact with red cells, or die. Cell death and cell growth/division essential for formation and maintenance of complex, asymmetrical morphology (cf. Fig. 2). $J(i j)=27,20 ; 16-, 8 ; 23,22,29,6(i=$ dark-gray, light-gray, red; $j=$ medium, $\ldots, i) ; \lambda=0.5, \tau=2, T=7$. (d) Intercalate and stretch. Stable differentiation by unequal cell division at twoand four-cell stage (blue, brown, light green). Further neighbor-based differentiation of blue in purple, of brown in dark-green and white, and of light-green in yellow. All cells are in three-state limit cycles. Morphogenesis without cell growth/division, and without apoptosis. Maximization of blue/brown contact layer by intercalation of green cells, which on contact with blue cells re-differentiate to brown. (Slow) engulfing of and pressing inwards into the light-green cell clump by brown and blue cells. "Gliders" rapidly traveling around the edge of the light-green cells consist of cells of the three cell lineages, see text. Final structure remains very dynamic. $\lambda=0.5, \tau=\infty, T=7$. (e) Intercalation and meristematic growth. Stable differentiation by unequal cell division at two- four-cell stage. Further neighbor-based induction in total 24 cell types throughout the development. The upper-right cell lineage (blue and yellow cells in 16-cell stage) is in a two-state attractor, all other cells are in a fixed-point attractor. Morphogenesis starts with elongation in two directions: up because of engulfing/intercalating "frustrated" by neighborhood-based redifferentiation [cf. Fig. 1(b)] and down because of successful intercalation of (part of the) upper-left cell lineage into the bottom/rear. This leads to (unlimited) meristematic growth in the upper part, and (limited) intercalary growth in the rear and invagination of red cells and yellow cells in the rear part. There are long-range interactions between these processes see Fig. 3(c). Cell lineages are also shown in Fig. 3(c). Evolutionary history of this critter is shown in Fig. $1 . \lambda=0.5, \tau=5 ; T=3$. 



A. Cell migration and Engulfing


B. Budding and Elongation

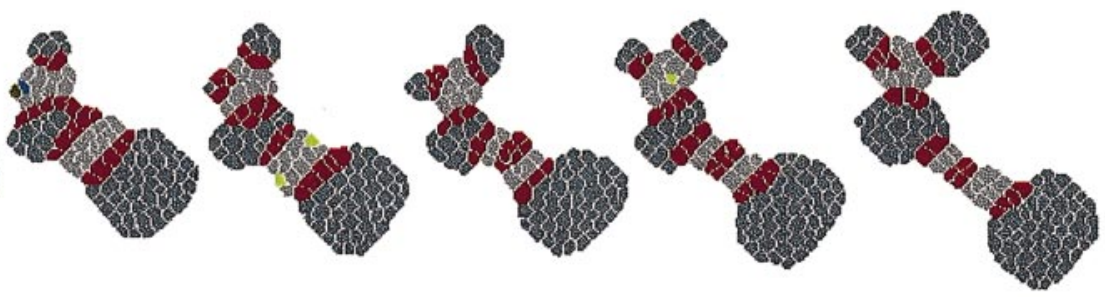

C. Cell Death and Redifferentiation
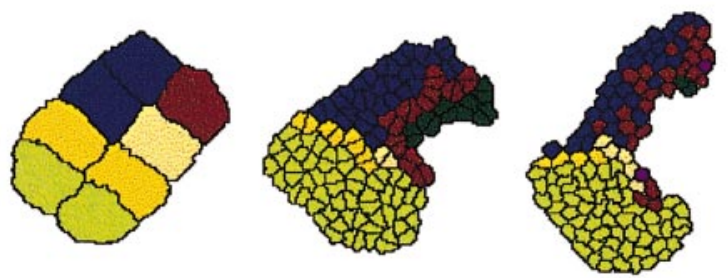

D. Intercalate and Stretch


E. Intercalation and Meristematic growth

FIG. 1. (Caption opposite). 




FIG. 3. (Caption opposite).
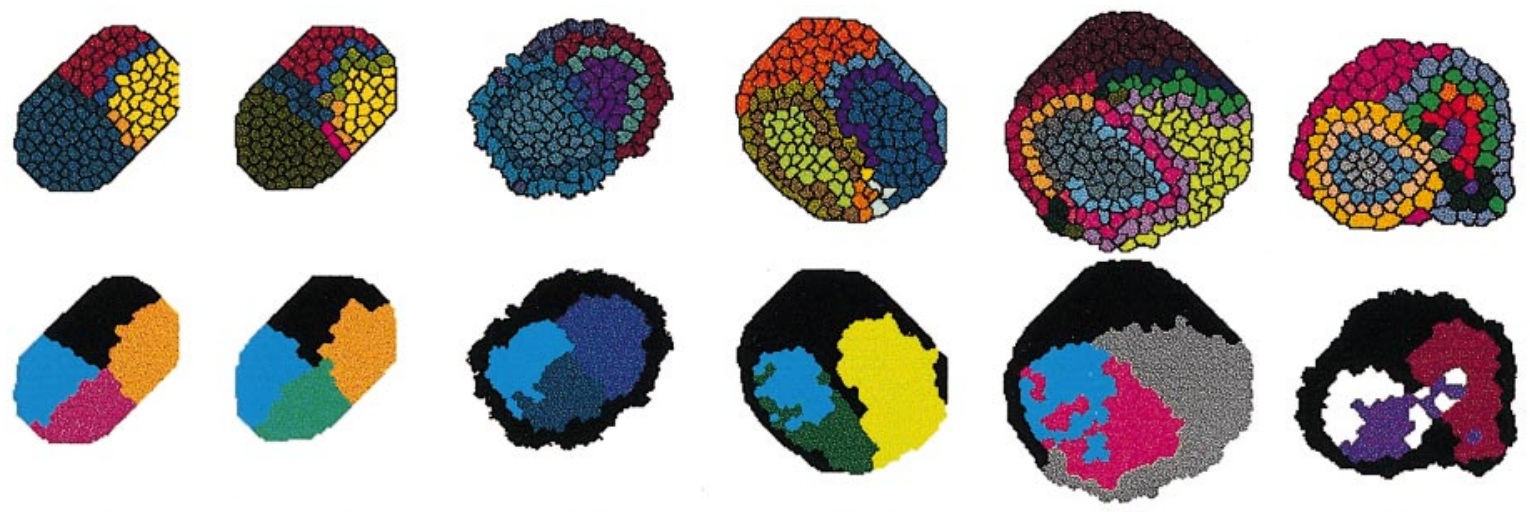

$E T=430$

$E T=518$

$E T=753$

$E T=950$

$E T=2143$

$E T=2709$
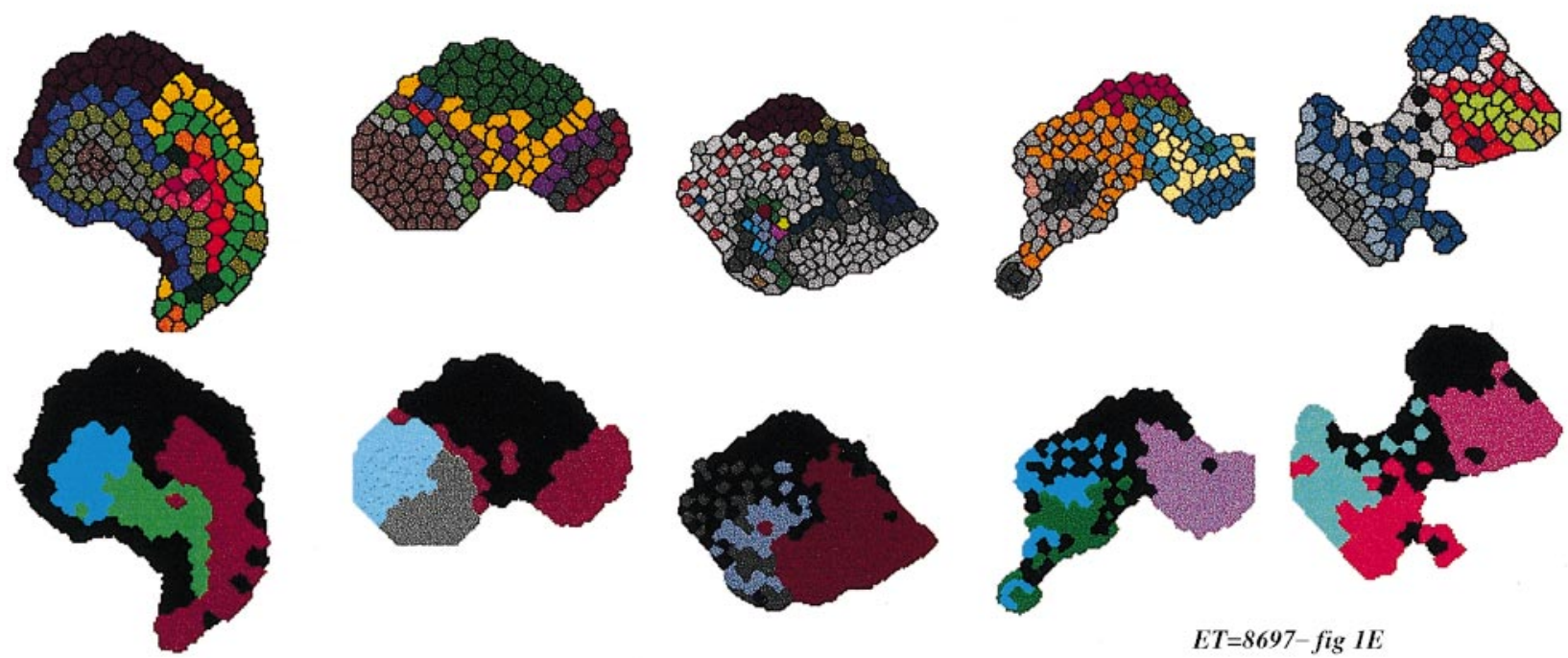

$E T=3259$

$E T=3272$

$E T=5308$

$E T=8697-f i g ~ I E$

$E T=6891$

$E T=8728$

FIG. 4. (Caption opposite). 
From these and other examples we conclude that:

- Initial stages are generally more sensitive to noise than later ones.

- Integrity of cell layers is ensured by neighborhood-dependent (re)differentiation. This is a strong pattern-preserving force.

- Therefore, the more complex cell differentiation leads to less sensitivity to noise.

In our simulations, we maximize cell differentiation over evolutionary time. For biotic systems robustness is an obvious requirement. The finding that increased cell differentiation leads to robustness, may play a role in reversed direction in biotic systems: the requirement for robustness of complex morphologies may lead evolutionarily to more complex gene regulation, and cell differentiation. Note that it is not feasible to do our simulations in the reversed order: the most robust solution is an undifferentiated blob!

\subsection{CELL- VS. NEIGHBORHOOD-BASED MEMORY AND MORPHOGENESIS}

Cell differentiation can be accomplished with various degrees of cellular "memory". Differentiation fully based on intra-cellular memory is caused by a signal leading to a neighborhoodindependent attractor basin. Conversely, the differentiation can be fully determined by the actual neighborhood, i.e. blocking cell signaling leads to de-differentiation but later re-establishing the signaling leads to the original differentiation pattern. In contrast, neighborhood-based memory is established by a sequence of neighborhood signals, is maintained by the neighborhood, and is lost when neighborhood signaling is blocked temporarily. These modes can of course be superimposed, leading to reversible further differentiation within memory-based attractor basins. All these modes are seen in our simulations, and play a different role in morphogenesis.

Neighborhood-based memory is least frequent, but it occurs in the complex morphogenesis of Fig. 1(c) and is responsible for the sensitivity to

FIG. 3. Examples of morphogenesis, alternatives and experiments. (a) Alternative patterns of engulfing. The morphogenesis directed by gene-regulation network of Fig. 1(a) is sensitive to small variations in the rate of cell migration. The two alternative patterns are shown here. Faster engulfing (slower apoptosis) leads to closure before the purple cells are "inside", and a hollow structure remains. Slower engulfing leads to absence of closure, and the formation of a "gut". (b) Suppression of intercalation. Intercalation of blue and gray cells is suppressed in the gene-network of Fig. 1(b) by setting $J$ (gray, blue) $=7$. Now the cell divisions at the "meristem" between gray and yellow cells produce new cells towards the periphery which become yellow. The resulting critter is shown colored according to gene-expression pattern, and according to number of cell divisions (see color-bar for coding): cell growth and division is localized on the two meristems. The evolved critter $[J$ (gray, blue) $=5]$ is also shown in the cell-division color code, and it is seen that cell growth and division is more distributed. (c) Cell growth, cell lineages and non-local interactions. Cell growth/division patterns are shown for (another individual of) the gene regulation network of Fig. 1(e) in a late stage. The top meristem is clearly seen. In the rear, cell divisions are more distributed. Initially, two well-defined growth zones were present, the top meristem, and the zone in which cells of the top intercalate to the rear. The latter growth is finite, and occurs on the (moving) migration zone. Moreover, cell division is increased further down by cell death of the red cell [see Fig. 1(d)]. The critter is also color-coded in terms of cell lineages (see four-cell stage for color code). The black cells are the most migratory, and cause much late redifferentiation of other cells. Non-local interactions of morphological features occur. The movement of the black cell lineage pulls along cells of the gold cell lineage downwards (V shape), and causes the separation of the top and bottom half of the critter by a "neck". Suppression of the intercalation of the black cells to the rear leads to the rightmost critter, which misses both these features. When intercalation is suppressed up to the 64-cell stage, the V shape develops but the "head" is joined to the rear like in the case of total suppression. The resulting critter resembles that shown in Fig. 4, ET5308, but has the "curl" in the top meristem, which is caused indirectly by single (pairs) of black cells among the golden cell lineage.

FIG. 4. An evolutionary history of morphogenesis. Stages in the evolutionary history which produced the critter of Fig. 1(e) are shown in two color codes: the gene expression patterns and the four cell lineages. Note the rotation relative to Figs. 1(e) and 3. The evolutionary dynamics is shown in Fig. 5. The upper panel shows the subsequent cell differentiation "inventions" and high-fitness critters are depicted. Cell growth/division occurs only in the later stages. The lower panel shows later evolutionary stages, when alternative morphologies occur in the "shadow" of the neutral path which maintains the intricate cell differentiation. These have much cell migration and therewith many gene expression patterns which are present only temporarily. Therefore they have lowered fitness. It is seen that morphological "inventions" occur in various combinations, so that mosaic-like evolution would be inferred at the morphological level, but not at the genome level. 


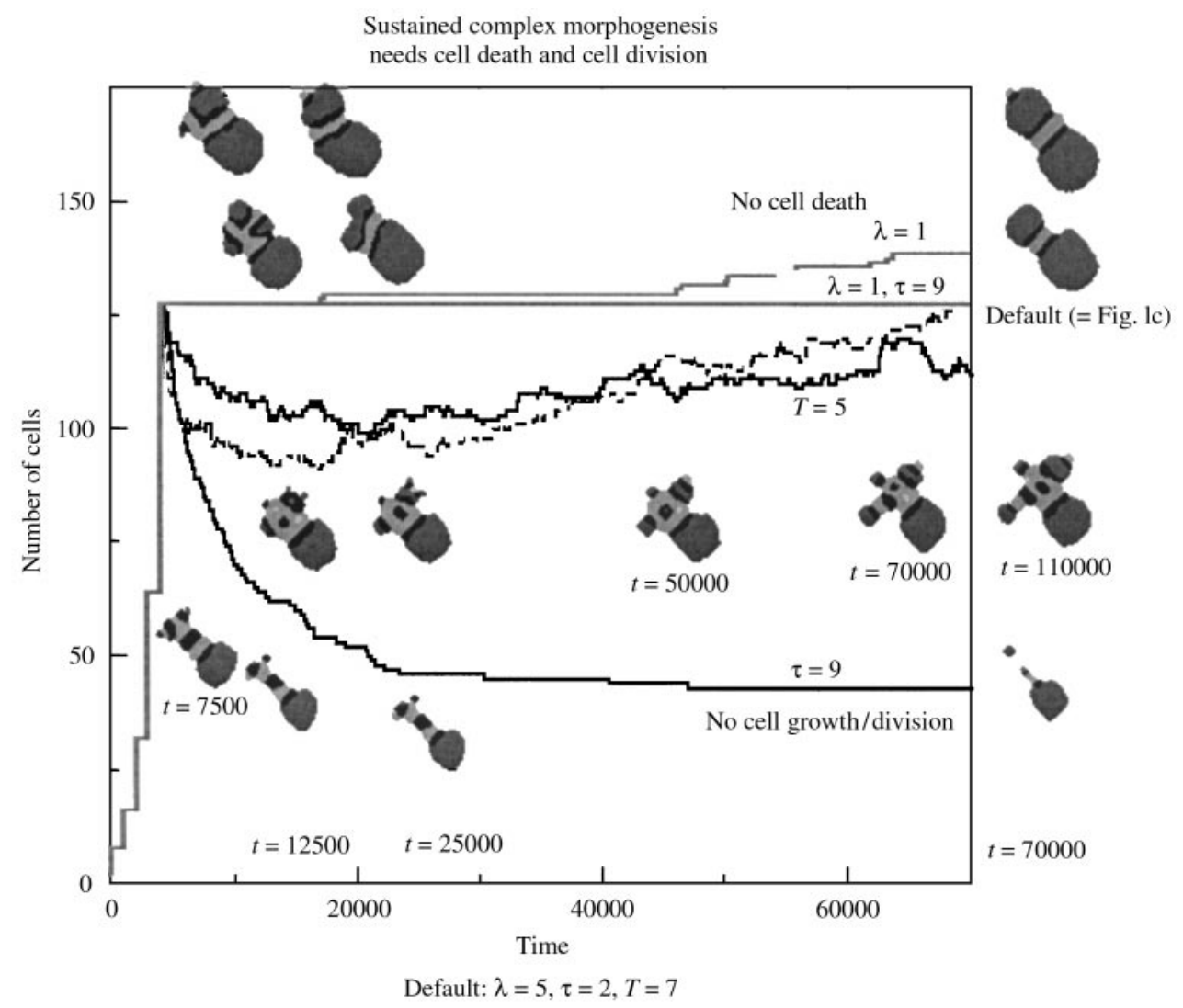

Fig. 2. Developmental dynamics. The figure shows the growth and development through time of critters with the gene regulation network of Fig. 1(c) (labeled "default") but different cell growth and cell death parameters. It shows that the cell (re)differentiation as dictated by the network maintains complex morphogenesis only in cooperation with cell growth and cell death.

noise in the initial conditions. Neighborhoodbased memory also occurs sometimes with respect to gene expression patterns which occur only late in development.

In most cases the networks configure themselves so that a stable genetic switch occurs to a new (set of) attractors in response to one or both maternal factors. This is mostly accomplished by a short loop in the internal gene regulation network, e.g. a self-reinforcing NOT-XOR node having a constant input which is switched momentarily by the maternal factor (chemically, such a transcription regulation can be accomplished by the formation of dimers).

Further differentiation is mostly entirely determined by the actual neighborhood (but maintaining the memory of the maternal signal). Redifferentiation occurs on changes of cell contact. This redifferentiation plays a crucial role in the described cases of morphogenesis. For example it maintains the stress zones leading to meristems and the elongation by budding and by lateral intercalation, and in regulation of the balance between apoptosis and cell division in Fig. 1(b, c, d, e). Only in the pseudo-gastrulation case reversal of the induced green cells to pink cells does not seem absolutely necessary for the complex engulfing (although it does occur). Intercalation of cells as seen in Fig. 1(b) and (d) and the resulting elongation is a mechanism that requires stable differentiation. As discussed in Section 3.1 these two modes have long-range interactions.

We conclude that the observed evolutionary preference of memory-less differentiation on top of permanent-cell-based differentiation is a powerful morphogenetic mechanism, due to the fact that neighborhood-dependent differentiation can automatically maintain local conditions and 
thus enhance pattern formation and contributes to the robustness of the patterns over time.

However, we suspect that this evolutionary preference also arises by the imposed constraint of fixed-point attractors (or short cycles) because cell-based memory needs cycles.

Our critters represent of course neither plants nor animals. For one thing they are two-dimensions. Plants are supposed to have much less cell-based memory than animals do. It is interesting to note that "animal-like" morphogenesis such as the gastrulation-like engulfing and the intercalation and stretching depend on stable differentiated cell lineages, whereas the "plant-like" morphogenesis due to meristematic growth depends on neighborhood-based redifferentiation of cells.

\subsection{INTERACTIONS AT ALL SCALES}

The model we use is defined on two scales: the local CA rules, which by copying states into neighboring sites change the shape of the modeled biotic cells, and the scale of these biotic cells comprising many (up to 80 in our experiments) cellular automata cells. The cell scale enters the $\mathrm{CA}$ rules via the volume-conservation term co-governing the probability of state copying at the scale of the CA sites. These two pre-defined scales lead to forces across the whole critter.

For example, the elongation in Fig. 1(b) is due to pulls in opposite directions at both ends of the critter. The pulls are generated by conflicting forces acting on single cells: a tendency of the gray cells to engulf the yellow ones, and the tendency to minimize the contact zone with them because of relatively high $J$. This leads to elongated gray cells cupping the yellow once and pushing them outwards. Such a conflict is eventually resolved, unless, as in the current model, it is self-maintaining by cell growth/division or redifferentiation. The way in which the conflict is resolved (maintained) can depend on non-local factors. In this case, the strong elongation of the stem occurs because of the intercalation of gray and blue cells. Without intercalation, a halterlike structure develops with an increasing number of yellow cells [Fig. 3(b)]. The long-range interactions are strikingly demonstrated when the stem breaks (as it does eventually when cell division is blocked): the stem is retracted very rapidly.

Another example of global effects of the behaviour of one type of cell on the behavior of other cells is demonstrated in Fig. 3(c): a blocking intercalation of cells to the rear leads to a global change in morphology in particular in the front of the critter. In fact, a critter with similar "pathological" morphology occurred during evolution (see Section 4).

We conclude that

- the two scale CA effectively implements longrange interactions;

- long-range interactions lead to a combinatorial explosion of morphologies formed by a limited number of local processes.

\subsection{TRANSIENTS, FRUSTRATION AND CELL GROWTH/DIVISION}

Asymmetric cell division, leading to differential gene expression, and hence to differential adhesion, generates an initial condition far from equilibrium. The transient to the minimization of $\mathrm{H}$ involves cell migration and shape changes, and long-range interactions conflicting locally with its minimization, but would, in a system of invariant cells, lead fairly rapidly to an equilibrium. However, in our model "frustration" arises because of conflicts between cell redifferentiation and cell adhesion. This may "condemn" cells to be neighbors despite relatively high $J$ [Fig. 1(c)], or may define a zone of cells "trying" to intercalate or engulf in between two layers of cells: success stops the "urge" of that cell but automatically generates another cell which takes its place [Fig. 1(b) and (d)]. Nevertheless, relaxation to (semi) blob-like critters occurs when no cell growth/division occurs. Cell growth (and cell death) are not externally imposed in our model, but occur only as a consequence of the forces created by the differential adhesion and cell differentiation: we do not have growth factors defined in the genome. Thus, growth occurs in zones of stress and death occurs in zones of squeeze. A priori one could expect this to lead to relaxation of these forces. In conjunction with the cell differentiation it does not, but maintains 
a non-equilibrium situation. Growth and shrinkage of cells are self-reinforcing processes in the model because of surface/volume ratio and curvature effects.

We conclude the following:

- The observed morphogenesis can be interpreted as maintenance of a transient.

- Surprising "coordination" between cell growth, cell death, cell migration and cell redifferentiation arises automatically within our model.

\section{Evolutionary Dynamics}

In this section we discuss the evolutionary dynamics in terms of some quantitative aspects, and in terms of the morphologies which are generated over evolutionary time. Next we discuss the occurrence of mosaic-like evolution at the morphological level while at the genomic level we observe linear, clockwise change. In connection with the latter observation we discuss determinism and randomness in evolution with a multilevel non-linear mapping between genotype and phenotype.

\subsection{NEUTRAL PATHS, AND STEPWISE EVOLUTION}

The evolutionary dynamics shows all the hallmarks known of evolution with a highly nonlinear mapping from genotype to phenotype and fitness. Such evolutionary dynamics is extensively studied e.g. for RNA, with a mapping from primary to secondary structure (see e.g. Huynen et al., 1995; Huynen, 1996; Fontana \& Schuster, 1998). For a general theory see van Nimwegen et al. (1997, 1999). Such evolution is characterized by extensive neutral paths on which the population "diffuses", and stepwise changes to a neutral path of higher fitness.

A specific example is presented in Fig. 5 which shows the overall evolutionary dynamics in terms of changes at the genomic level, and on the "structural" level, i.e. the shape of the functional gene regulation network. The figure shows the following:

- The stepwise increase of median fitness of the population, and a large variation in fitness within the population. The variation is large because of the major impact one mutation might have [Fig. 5(c)].

- Molecular clock with respect to cumulative genomic change [Fig. 5(a)].

- Slowdown of change in functional gene regulation network [Fig. 5(b)]. The functional network is determined by pruning all constant nodes and non-informative connections. One point mutation can generate extensive functional change as well as no functional change.

- Conservation of network properties over relatively long periods of time [Fig. 5(d)], but fitness is not easily related to any network property. Sometimes, a network change can become important for fitness only after other changes.

- During evolution we observe an increase in cellular signaling and a decrease in non-forcing functions (XOR) and a decrease of the number of "leaves" (i.e. downstream genes) [Fig. 5(c) and (d)].

\subsection{EVOLUTION OF MORPHOLOGIES: AN EXAMPLE EVOLUTIONARY HISTORY}

Figure 4 shows a selection of morpho-types through evolutionary time, again of the same evolutionary history as discussed in the previous section, which leads to the morphogenesis shown in Fig. 1(e). The stages were selected to portray the variety of morphologies. These are shown in two color codes: the cell "types" (gene expression patterns) (upper rows) and cell lineages, i.e. the offspring of each of the four first cells. The latter representation facilitates the visualization of the way the critters develop.

In the early stages of evolution (up to ET 2143), successive new neighborhood-based differentiation signals are used on top of the differentiation into three stably differentiated cell lineages due to the maternal signals. The first sample shows no cell migration and no cell growth or cell death. It uses one inter-cellular signal which causes neighborhood-dependent differentiation between the cell lineages. The second sample has two signals, which leads to two differentiated layers between the three cell lineages. Moreover, very slow cell migration occurs: the black cell lineage squeezes in between the others. Still, there is no cell 
Evolutionary dynamics
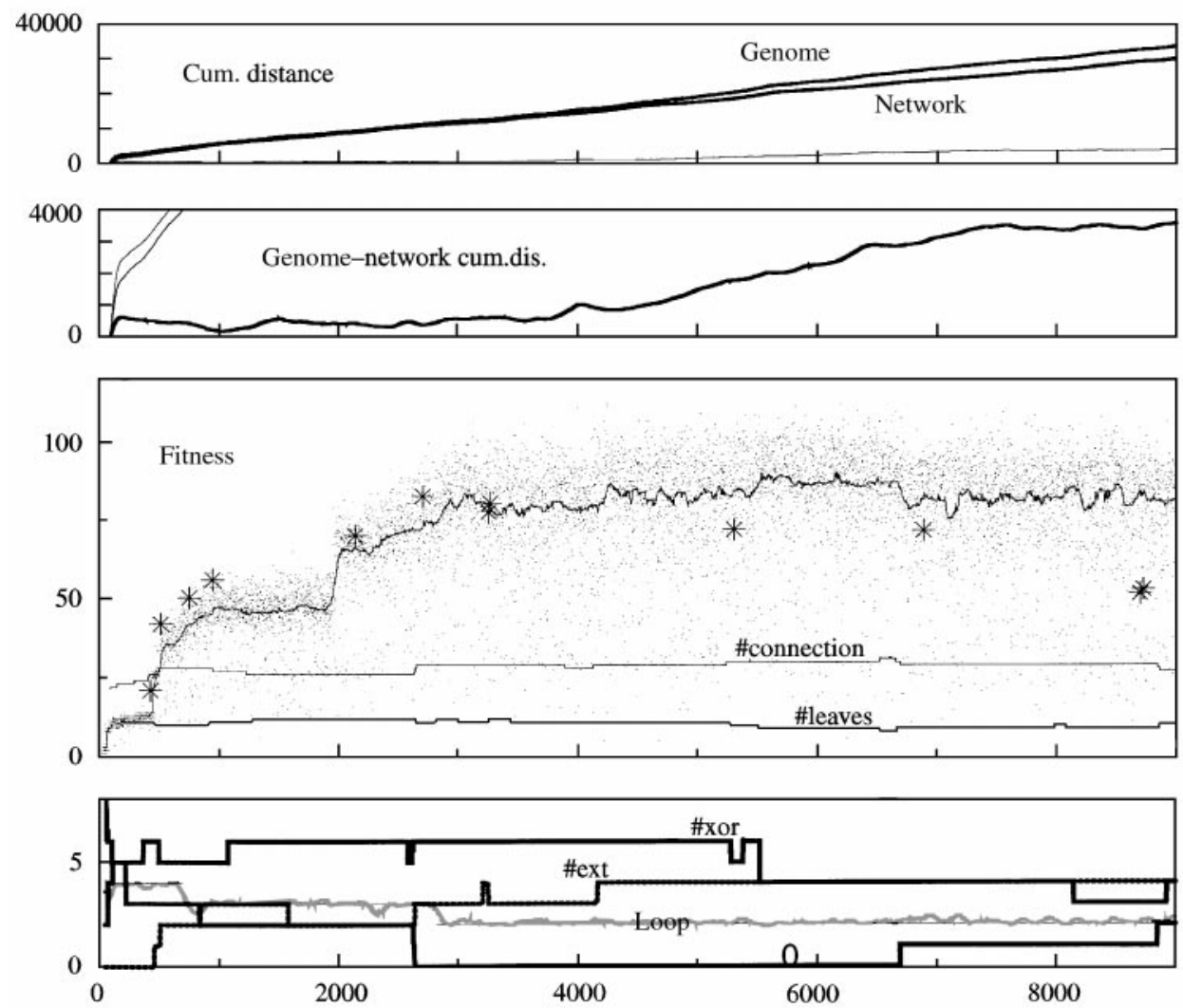

FIG. 5. The evolutionary dynamics through time. Time is measured in terms of the number of critters generated, and thus uniquely identifies a critter. Upper panel: length of trajectory traveled through genome space. This is calculated as the cumulative distance between the centroids of a population of ten critters, 100 time-steps apart. Upper line distance between genomes, i.e. number of differences in the genomes. Lower line the distance is measured as the number of differences in the functional gene regulation network. Here the network is reduced to functional link (i.e. those which influence the gene expression). Note that one mutation can cause multiple changes in the functional network. Second from above: the difference between the length of the trajectory traveled through genome space and through network space. Initially, mutations cause on average the same amount of change in the genome and in the functional network. Once the full cell differentiation pattern is reached, the change in the functional network slows down. Middle: fitness over time. The dots give the fitness of each evolved critter: single mutations cause major changes in fitness. The thick line is the running median fitness over 100 time steps. The stars are the samples depicted in Fig. 4. The lower lines depict some network properties: upper line the number of network connections, lower line the number of downstream genes, which do not regulate any other gene(s). Bottom. Network properties [shown as running median (200 time steps)]. XOR: the number of non-forcing functions in the network, i.e. the boolean functions which depend on all inputs, regardless of the value of the inputs. For two-connected networks these are "exclusive or" and its negation. Their number declines and therewith tends to increase mutational stability of the network. Loop: number of nodes in network loops. Loops are important for stable cell-based memory. Here it is not functional and declines through drift. \# ext: number of nodes connected to external signals. Initially increases, later drifts, but initial neutral changes become essential later on. 0: number of genes which are never expressed. First declines sharply, and becomes zero. Slight fitness decrease later on is associated with loss of expression of gene(s).

growth/death. The third sample shows rapid engulfing of the entire critter by the black cell lineage. These cells now grow and divide leading to an ever thicker outer layer. The next sample combines both types of migration: the black cell lineage now engulfs around and squeezes in between the other cell lineages, although it does so very slowly (engulfing is not completed in the developmental stage shown). The squeezing leads to three differentiated cell layers between the two "compartments". The next stage (ET2143) has reached the next fitness plateau. Indeed it has the main cell differentiation pattern which will persist for a long time, and which will give rise to an 
explosion of morphologies. Curiously, no conspicuous (quantitative) network changes accompany this major evolutionary step. Two cell lines now engulf, the one which did so previously from the "rear", and another one (which expands strongly by cell growth/division) from the front. Engulfing stops when they meet. Cell movement is even more intricate in the next stage where sub-types of all lineages participate in cell migration of layers of cells. In the process, new cell contacts are established and therewith new gene expression patterns. The next stage (ET3259) is very similar to the previous one, but slower engulfing of the "black" cell lineage, and increased engulfing and redifferentiation between neighborhood-based differentiated cells of the "red" cell lineage now cause a directional (downward) outgrowth of the critter. The development looks like a slowly advancing "slug". These critters form the "trunk" of the evolutionary tree, and subsequent critters on the trunk show many subtle and less subtle variations of this and the previous critters. We further discuss some patterns which appear in the side-branches.

Almost simultaneously with the previous sample (ET3272) the next sample occurs, as a (high fitness) side-branch of the evolutionary tree. Although the early differentiation pattern is still similar, it is morphologically quite different. Here the black cell line does not engulf, but squeezes between the front and the rear (or more precisely between the front and the front-derived cell layer which differentiates due to contact with the rear part) and expands. The critter shows the first occurrence for the later often reoccurring sharp bend at the top. Next, one more external signal is added to the network. Morphologically, the next invention is the intercalation instead of engulfing of the black cell line among the rear cells. This feature also occurs in the critter of Fig. 1(e), and is reinvented repeatedly in the side-branches of the evolutionary tree. ET5308 is in fact similar to the "pathology" shown of the critter of Fig. 1(e) when intercalation is inhibited in early developmental stages. Moreover, critter ET 6891 is essentially the same as the one of Fig. 1(e), which itself appeared as ET8697. They combine intercalation in the rear with a strong growth meristem in the front. They are both fairly low-fitness side-branches of the evolutionary tree, and the morphology is reinvented repeatedly (6 times in this evolutionary history). The fitness is low because cell differentiation is measured over a short period of time, while in these critters many differentiation patterns occur only during short periods in the development. Finally, we show one more sidebranch which shows another combination of limited intercalation to the rear, and limited expansion in the front.

\subsection{MOSAIC EVOLUTION}

Thus, we see that the explosion of morphologies arise as a result of different combinations of cell migration and cell growth properties. Notwithstanding the progressive change at the genomic and the network level, we see a reoccurrence of similar morphogenetic mechanisms at different moments in evolution. We used small population sizes (20) because of computer limitations, and the phylogenetic tree has a main stem with only short side-branches. As mentioned, the main stem of the phylogenetic tree morphologies are dominated by ones similar to those of ET2709 and ET3259, i.e. an intricate rotational cell migration pattern and directional elongation. The additional features, e.g. intercalation of the black cell lineage and therewith elongation of the "rear", and meristemic growth in the "front", and invagination are "reinvented" several times, and occur in different combinations (note that no recombination is used at the genomic level). They are in fact caused by mutations leading to loss of network connections, and to invariant expression of genes. Thus, at the morphological level a mosaic-like evolution appears. Apparently, these alternative morphologies occur in the so-called "shadow" of the neutral path through fitness space traveled by the population. Such a shadow was also identified in the RNA landscapes, but there often contain rather similar secondary structures to the conserved one (Huynen, 1997). Here the similarity is not obvious although present at a deeper level. Building phylogenetic trees on morphological features certainly would be a nuisance if evolution exploits such alternatives which remain permanently "available" because of some conserved property at the genomic level. 


\subsection{DETERMINISM AND RANDOMNESS IN EVOLUTION}

Reinvention of apparently intricate mechanisms bears on questions of randomness and determinism is evolution. In our simulations, we see that every evolutionary run is unique and depends sensitively on the seed of the pseudorandom generator. However, when the population attained after some time (e.g. after ET 2000) is re-initiated with another seed of the pseudorandom generator the evolution unfolds in a strikingly similar manner. At the genomic level the evolution branches off in two different directions, and the regulation networks differ. The main stem morphologies are similar, but certainly not identical. Nevertheless, very similar morphologies may occur on the side-branch. In our simulations, they are often caused by loss mutations on the genomic level, but, before knowing this, we selected them as interesting cases because of their intricate morphological development.

In biotic systems striking cases of parallel evolution have been reported. For example, the morphological variation of Anolis lizards is similar on different islands, but has evolved anew on each of them starting from different points, as can be observed by looking at the genomic level (Losos et al., 1998). A possible explanation could be that these morphologies occur in the "shadow" of the evolutionary neutral path of these lizards as measured in some other dimension. In the case of the lizards this shadow has been exploited to occupy the available niches on the islands.

\section{Discussion and Conclusions}

A fundamental question in evolutionary theory is to what evolved features are "frozen accidents", dynamically relatively "easy" (and generic) cases, or have primarily evolved to meet specific environmental demands (and/or combination thereof). Although multi-cellularity has evolved several times in biotic systems (e.g. plants and animals) the number of times is far too small to even approach this question from data on evolved biotic systems only.

The studies presented here aim to shed some light on this question, with respect to basic morphogenetic mechanisms. To this end, we explored what are relatively generic cases for cellular systems with differential adhesion and dynamic cell differentiation. We have found that the interplay between these processes is sufficient to generate and maintain non-blob-like structures. Moreover, the mechanisms for doing this appear to be similar to well-known mechanisms in biotic systems, e.g. engulfment, growth meristem, intercalary growth, and intercalate and stretch. With these morphogenetic mechanisms a combinatorial explosion of shapes can be generated, and the pseudo-isomorphic growth and development are strikingly "life-like".

One should note that our results go beyond: "these types of morphogenesis are possible with cell adhesion and cell differentiation". We have shown that they are "relatively generic" for such systems, and arise even if they are "good for nothing", and without any tuning of parameters. Methodologically, the main contribution of this paper is the demonstration that abstract theoretical models can uncover "relatively generic" features, or as we called it earlier (Hogeweg, 1998) "non-generic-generic phenomena". They are nongeneric because most cases of cellular adhesion and cellular differentiation will simply form blobs. However, recognizing that cell differentiation is a pre-requisite, and zooming in on cases meeting this pre-requisite, morphogenesis by the above-mentioned mechanisms is a frequent sideeffect in our adhesion-driven system.

Previous modeling effort for biological development has emphasized the role of gradients of morphogens. Although diffusion is a very simple process in physical systems in the very heterogeneous environment of cellular systems, quite elaborate mechanisms of receptor-mediated processes are needed to produce a gradient (Kerzberg 1996; Kerzberg \& Wolpert, 1998). Similarly, the physical simple process differential adhesion is accomplished in cellular systems by diverse and intricately regulated receptorbased processes. The use of "adhesion" in our models should be seen as a shortcut similar to the use of "diffusion" in gradient and reactiondiffusion-based models of biological development.

In this paper, we explored pattern formation without concentration gradients, allowing only for binary gene expression differences and 
cell contact interactions. It turns out that in the initial stages of development a gradient-like pattern of gene expression is often formed. The gradients we find are similar to those found in the expression of homeotic-type genes in that not the amount of one morphogen makes the gradient, which is impossible in our model, but rather it is defined by overlapping ranges of expression of genes.

Nevertheless, an interesting extension of the model will be the inclusion of concentration gradient, and study what additional morphogenetic potential and/or stabilization occurs in such systems. The finding (Hobmayer et al., 1996; Holstein, 1999) that the gradient-based Wnt signalling pathway, including its close coupling to the cadherin/catenin adhesion/transcription regulating system is already fully present and active in the development of Cnidaria, i.e. very primitive metazoans, make it even more interesting to study systems in which gradients, cell adhesion and cell differentiation are closely coupled. Other possible extensions are, for example, the inclusion of "active" cell movement (e.g. chemotaxis), and cell shape changes [see e.g. the early model of Odell et al. (1981), and the recent model of Kerzberg \& Changeux (1998) which focus on these] as well as cell growth and cell death triggered from within, i.e. by gene regulation rather than by the adhesion forces which cause these processes in the present model. (Note that although stretch/squeeze triggered cell growth/death has been demonstrated experimentally appears to have a wider repertoire (see e.g. Gumbiner, 1996; Howe, 1998; Marrs \& Nelson, 1996; Giancotti, 1997) However, cell adhesion and cell differentiation should be basic ingredients in such extended models as well. Interpretation of the results of such more complex models needs as baseline the insights obtained from the simpler system studied in this paper. Note that in the system studied here the orchestration between cell movement, cell growth, cell death, and cell (re)differentiation happens automatically due to the fact that all these processes are linked via differential adhesion. Orchestration of the different processes in the extended models seems harder, and possibly can best be achieved by building on this adhesion-based automatic coordination.
Finally, remember the famous anecdote which relates that Turing, upon praise of his "turing patterns" said: "the stripes are easy but what about the horse part". On the basis of our studies we can extend this to: "stripes and morphogenesis are "easy", but obviously we should keep in mind that there is more to a horse.

This work was initiated in collaboration with Roeland Merks. I am much indebted to him for the great amount of work he did in setting up and programming the combined developmental and evolutionary model. I also thank Nick Savill and Stan Marée for insightful discussions. They use(d) the developmental model in combination with long-range signalling for modeling "real" morphogenesis of Dictyostelium. Finally, I thank Ben Hesper for long-term inspiration and support.

\section{REFERENCES}

Chen, C. S. Mrksich, M., Huang, S., Whitesides, G. M. \& INGBER, D. E. (1997). Geometric control of cell life and death. Science 286, 1425-1428.

FontanA, W. \& Schuster, P. (1998). Continuity in evolution: on the nature of transitions. Science 280, 1451-1455.

Furusawa, C. \& KaneKo, K. (1998a). Emergence of multicellular organisms with dynamic differentiation and spatial pattern. Artif. Life 4, 79-93.

Furusawa, C. \& KANEKo, K. (1998b). Emergence of rules in cell society: differentiation, hierarchy, and stability. Bull. Math. Biol. 60, 659-687.

GiAnCOTTI, F. G. (1997). Integrin signalling: specificity and control of cell survival and cell cycle progression. Curr. Opin. Cell Biol. 9, 691-700.

Glazier, J. A. \& Graner, F. (1993). Simulation of the differential driven rearrangement of biological cells. Phys. Rev. E. 47, 2128-21254.

Gumbiner, B. M. (1996). Cell adhesion: the molecular basis of tissue architecture and morphogenesis. Cell 84, 345-357.

Hobmayer, E., Hatta, M., Fischer, R., Fujisawa, T., Holstein, T. W. \& Sugiyama, T. (1996). Identification of a Hydra homologue of the beta-catenin/plakoglobin/ armadillo gene family. Gene 172, 155-159.

HOGEWEG, P. (1998). On searching generic properties of non generic phenomena: an approach to bioinformatic theory formation. In: Artificial Life VI (Adami, C., Belew, R. K., Kitano, H. \& Taylor, C. E., eds), pp. 285-294. Cambridge: MIT Press.

HOLSTEIN, T. (1999). Lecture at NATO workshop on the origins of multicellularity. Budapest.

Holy, T. E., Dogterom, M., Yurke, B. \& Leibler, S. (1997). Assembly and positioning of microtubule asters in microfabricated chambers. Proc. Natl. Acad. Sci. U.S.A. 94, 6228-6231.

Howe, A., Aplin, A. E., Alahari, S. K. \& Juliano, R. L. (1998). Integrin signaling and cell growth control. Curr. Opin. Cell Biol. 10, 220-231. 
HuYNEN, M. A. (1996). Exploring phenotype space through neutral evolution. J. Mol. Evol. 43, 165-169.

Huynen, M. A., Stadler, P. F. \& Fontana, W. (1996). Smoothness within ruggedness: the role of neutrality in adaptation. Proc. Natl. Acad. Sci. U.S.A. 93, 397-401.

JACKSON, E. R., JOHnSON, D. \& NASH, W. G. (1986). Gene networks in development. J. theor. Biol. 119, 379-396.

JiAng, Y., LeVine, H. \& GlaZIER, (1998). Possible cooperation of differential adhesion and chemotaxis in mound formation of dictyostelium. Biophys. J. 75, 2615-2625.

KANEKO, K. \& YOMO, T. (1997). Isologous diversification: a theory of cell differentiation. Bull. Math. Biol. 59, 139-196.

KAUfFMAN, S. A. (1969). Metabolic stability and epigenesis in randomly constructed genetic nets. J. theor. Biol. 22, 437-467.

KERZBERG, M. (1996). Accurate reading of morphogen concentrations by nuclear receptors: a formal model. J. theor. Biol. 183, 95-104.

KerzberG, M. \& Wolpert, L. (1998). Mechanisms for positional signalling by morphogen transport: a theoretical study. J. theor. Biol. 191, 103-114.

Kerzberg, M. \& Changeux, J. P. (1998). A simple molecular model of neurulation. BioEssays 20, 758-769.

Losos, J. B., Jackman, R., Larson, A. De Queiroz, K. \& RODRGUEZ-SCHETTINO, L. (1998). Contingency and determinism in replicated adaptive radiations of island lizards. Science 279, 2115-2118.

Marée, A. F. M., PAnfilov, A. V. \& Hogeweg, P. (1999). Migration and thermotaxis of Dictyostelium discoideum slugs, a model study. J. theor. Biol. (in press).
Marrs, J. A. \& Nelson, W. J. (1996). Cadherin cell adhesion molecules in differentiation and embryogenesis. Int. Rev. Cytol. 165, 159-205.

Monier-Gavelle, F. \& Duband, J. L. (1997). Cross talk between adhesion molecules: control of N-cadherin activity by intracellular signals elicited by betal and beta 3 integrins in migrating neural crest cells. J. Cell. Biol. 137, 1663-1681.

van Nimwegen, E., Crutchfield, J. P. \& Mitchell, M. (1997). Finite populations induce metastability in evolutionary search. Phys. Lett. A 229, 144-150.

van Nimwegen, E., CRutchfield, J. P. \& Mitchel, M. I. (1999). Statistical dynamics of the royal road genetic algorithm. Theoret. Comput. Sci. (special issue on Evolutionary Comput.) in press. Santa Fe Institure working paper 9704-035.

Odell, G. M., Oster, G., Alberch, P. \& Burnside, B. (1981). The mechanical basis of morphogenesis. I. Epithelial folding and invagination. Dev. Biol. 85, 446-462.

RuOSLAHTI, E. (1997). Stretching is good for a cell. Science 276, 1345-1346.

Savill, N. J. \& Hogeweg, P. (1997a). Modeling morphogenesis: from single cells to crawling slugs. J. theor. Biol. 184, 229-235.

SAvill, N. J. \& Hogeweg, P. (1997b). A cellular automata approach for modeling cell-cell interactions. In: Dynamics of Cell and Tissue Motion (Alt, A., Deutsch, A. \& Dunn, G., eds). Basel: Birkhauser Verlag.

SteInBERG, M. S. \& TAKeICHI, M. (1994). Experimental specification of cell sorting, tissue spreading and specific spatial patterning by quantitative differences in cadherin expression. Proc. Natl. Acad. Sci. 91, 206-209. 